\title{
ФАРМАЦЕВТИЧЕСКАЯ ХИМИЯ
}

УДК 615.84:616.012

DOI: https://doi.org/10.52540/2074-9457.2021.3.64

Е. А. Дикусар ${ }^{1}$, А. Л. Пушкарчук ${ }^{1}$, Т. В. Безъязычная ${ }^{1}$, Е. А. Акишина ${ }^{1}$, А. Г. Солдатов ${ }^{2}$, С. А. Кутень ${ }^{3}$ С. Г. Стёпин ${ }^{4}$, А. П. Низовцев ${ }^{5}$, С. Я. Килин Л. Ф. Бабичев ${ }^{6}$ В. И. Поткин ${ }^{1}$

\section{ПЕРСПЕКТИВЫ СОЗДАНИЯ РАДОНОСОДЕРЖАЩИХ АГЕНТОВ РАДИОНУКЛИДНОЙ ТЕРАПИИ}

\begin{abstract}
${ }^{1}$ Институт физико-органической химии НАН Беларуси, г. Минск, Республика Беларусь ${ }^{2}$ Научно-практический центр по материаловедению НАН Беларуси, г. Минск, Республика Беларусь

${ }^{3}$ Институт ядерных проблем БГУ, г. Минск, Республика Беларусь ${ }^{4}$ Витебский государственный ордена Дружбы народов медицинский университет, г. Витебск, Республика Беларусь

${ }^{5}$ Институт физики им. Б. И. Степанова НАН Беларуси, г. Минск, Республика Беларусь бОбъединенный институт энергетических и ядерных исследований Сосны НАН Беларуси, д. Прилесье, Минский р-н, Республика Беларусь
\end{abstract}

Одним из методов лечения онкологических заболеваний является использование короткоживущих радионуклидов. При этом сущеествующие риски интоксикации и радиоактивного заражения можно исключить, помещая короткоживущий изотоп радона в прочную фуллереновую оболочку. Для повышения эффективности этих препаратов перспективным является введение структурных фрагментов аминокислот, пептидов, стероидных гормонов, нуклеиновых оснований или гетероциклических соединений, способных накапливаться именно в опухолевых, а не в здоровых тканях организма. В статье представлены данные по квантово-химическому моделированию электронной структуры и термодинамической устойчивости фуллереновых радонсодержащчих агентов радионуклидной терапии на примере модельного кортизонофуллеренолового биоконъюгата.

Ключевые слова: DFT-моделирование, изотоп радона, кортизон, фуллереноловые кластеры, агенты терапии опухолевых новообразований.

\section{ВВЕДЕНИЕ}

Для лечения онкологических новообразований обычно применяют хирургические методы, химиотерапию или лучевую терапию, а в изотопной медицине - вводят в опухоль короткоживущие радионуклиды, например: ${ }^{59} \mathrm{Fe},{ }^{90} \mathrm{Y},{ }^{95} \mathrm{Zr}$, ${ }^{99 \mathrm{~m}} \mathrm{Tc},{ }^{106} \mathrm{Ru},{ }^{114 *} \mathrm{In}$, ${ }^{147} \mathrm{Eu},{ }^{148} \mathrm{Eu},{ }^{155} \mathrm{Eu},{ }^{170} \mathrm{Tm},{ }^{188} \mathrm{Re},{ }^{210} \mathrm{Po},{ }^{222} \mathrm{Rn}$, ${ }^{230} \mathrm{U},{ }^{237} \mathrm{Pu},{ }^{240} \mathrm{Cm},{ }^{241} \mathrm{Cm},{ }^{252} \mathrm{Cf},{ }^{253} \mathrm{Es}$. Короткоживущие радионуклиды считаются крайне перспективными радиофармацевтическими средствами для лечения различных видов онкозаболеваний, в том числе и на их последней, уже терминальной стадии. При распаде они испускают альфа-частицы, которые уничтожают раковые клетки, не задевая здоровые. Однако при их распаде часто образуются токсичные изотопы, которые накапливаются в печени, почках, селезенке и других органах, что сдержива- ет широкое применение радионуклидов в клинической практике. В последние годы идет активная разработка специальных наноконтейнеров для адресной доставки радионуклидов непосредственно в опухолевые ткани с целью их уничтожения [1-3].

Одним из перспективных направлений борьбы со злокачественными новообразованиями может быть использование радиоактивного короткоживущего природного изотопа радона [4], инкапсулированного во внутреннюю полость фуллеренола, ковалентно связанного с биологически активными компонентами (фрагментами аминокислот, пептидов, стероидных гормонов, нуклеиновых оснований или гетероциклических соединений, способных накапливаться именно в опухолевых, а не в здоровых и неповрежденных тканях организма) [4-22]. Изотоп радона $\left({ }^{222} \mathrm{Rn}\right)$, имеющий период 
полураспада всего около 3,82 суток, при самопроизвольном радиоактивном распаде испускает альфа-частицы с энергией 5,59 МэВ, которые и должны уничтожать раковые клетки опухоли [4].

Сырьевой базой для получения чистого радона (методы его добычи путем экстракции, выделения и очистки хорошо известны и в настоящее время детально разработаны [4]) могут служить грунтовые воды радоновых источников. В Беларуси на таких радоновых источниках, расположенных в Дятловском районе Гродненской области, еще с начала 80-х годов действуют санатории «Радон» и «Альфа Радон», использующие радоновые ванны для облегчения хронических заболеваний, боли и усталости [23].

Целью данной работы являлась оценка возможности получения фуллереноловых радоносодержащих агентов радиационной терапии на примере модельного кортизонофуллеренолового биоконьюгата.

\section{МАТЕРИАЛЫ И МЕТОДЫ}

Объектами исследования были исходные компоненты для построения методом квантово-химического моделирования кортизонофуллереноловых кластеров: кортизон 1, фуллеренол 2, образующаяся в результате реакции конденсации вода 3 . Из смоделированного кортизонофуллеренолового кластера 4 и радона 5 строили радонсодержащие кластеры: 6 - с атомом радона снаружи кластера, 7 - с атомом радона внутри кластера, 8 - с двумя атомами радона, расположенными снаружи и внутри кластера.

Расчеты параметров изучаемых соединений проведены с использованием теории DFT/BP86/RI/def2-SVP/def2/J/D3 с помощью программного пакета ORCA [24] на суперкомпьютере «СКИФ-500» в Объединенном институте энергетических и ядерных исследований - Сосны.

В процессе расчетов проводили оптимизацию всех геометрических параметров кластеров до достижения минимумов полных электронных энергий. Тепловой эффект получения кластера 4 из кортизона 1 и симметричного фуллеренола 2 рассчитывали по формуле (1) [25, 26]:

$$
\Delta E_{\text {Int.(4) }}=\left[E_{(\mathbf{4})}+E_{(\mathbf{3})}\right]-\left[E_{(\mathbf{2})}+E_{(\mathbf{1})}\right],
$$

где $\Delta E_{\text {Int.(4) }}$ - тепловой эффект получения кластера $\mathbf{4}$,

$E_{(\mathbf{1})}-$ полная энергия кортизона $\mathbf{1}$,

$E_{(\mathbf{2})}^{(1)}$ полная энергия фуллеренола 2,

$E_{(\mathbf{3})}^{(2)}$ - полная энергия воды $\mathbf{3}$, pa 4.

$E_{(4)}^{(3)}$ - полная энергия «пустого» класте-

Квантово-химические расчеты энергий взаимодействия $\left(\Delta E_{I n t .}\right)$ кластера 4 с радоном 5, приводящие к образованию кластеров 6-8, проводили по формулам (2) и (3) $[25,26]$ :

$$
\begin{aligned}
& \Delta E_{\text {Int. (6, 7) }}=E_{(\mathbf{6}, 7)}-\left[E_{(\mathbf{4})}+E_{(\mathbf{5})}\right], \\
& \Delta E_{\text {Int. (8) }}=E_{(\mathbf{8})}-\left[E_{(\mathbf{4})}+2 E_{(\mathbf{5})}\right],
\end{aligned}
$$

где $\Delta E_{\text {Int.(6,7) }}$ - тепловой эффект взаимодействия для кластеров 6 и 7 ,

$\Delta E_{\text {Int.(8) }}$ - тепловой эффект взаимодействия для кластера $\mathbf{8}$, pa 4 ,

$E_{(4)}$ - полная энергия «пустого» класте-

$E_{(5)}-$ полная энергия атома радона $\left({ }^{222} \mathrm{Rn}\right) \mathbf{5}$,

$E_{(6)}$ - полная энергия кластера с атомом ${ }^{222} \mathrm{Rn}$ снаружи 6,

$E_{(7)}$ - полная энергия кластера с атомом ${ }^{222} \mathrm{Rn}$ внутри 7,

$E_{(\mathbf{8})}$ - полная энергия кластера с двумя атомами ${ }^{222} \mathrm{Rn}$ как внутри, так и снаружи 8.

Для оценки реакционной способности и, соответственно, биологической активности в работе использовали методологию теории граничных молекулярных орбиталей (ГМО), разработанную К. Фукуи [27]. Величины $\Delta E_{\text {гмо }}$, которые являются ключевыми характеристиками молекул в теории ГМО, рассчитывали по формулам (4) и (5):

$$
\begin{gathered}
\Delta E_{\text {ГМО }}=\left|\left[E_{\text {взмо }}-E_{\text {НВМо }}\right]\right|, \\
\Delta E_{\text {ГМО(мол.) }}=\Delta E_{\text {ГМО }} \cdot 6,022140857(74) \cdot 10^{23},
\end{gathered}
$$

где $\Delta E_{\Gamma м о}-$ разность энергий верхней занятой (B3МО) и нижней вакантной (HВMO) атомных или молекулярных орбиталей соответственно.

\section{РЕЗУЛЬТАТЫ И ОБСУЖДЕНИЕ}

Процесс построения кластера $(8 S, 9 S, 10 R, 13 S, 14 S, 17 R)$-17-гидрокси-17- 
(2-фуллеренол[ $\left.\mathrm{C}_{60}(\mathrm{OH})_{23}\right]$ оксиацетил)10,13 -диметил-1,2,6,7,8,9,12,14,15,16декагидроцикло-пента[a]фенантрен -3,11-диона) $\mathrm{C}_{81} \mathrm{H}_{50} \mathrm{O}_{28} 4$, который является ковалентным производным симметричного тетраэйкозофуллеренола $\mathrm{C}_{60}(\mathrm{OH})_{24} 2$ [28, 29] и кортизона $(8 S, 9 S, 10 R, 13 S, 14 S, 17 R)$ -
17 -гидрокси-1 7-(2-гидроксиацетил)10,13 -диметил-1,2,6,7,8,9,12,14,15,16декагидроциклопента[ $a]$ фенантрен-3,11дион) $\mathrm{C}_{21} \mathrm{H}_{28} \mathrm{O}_{5} \mathrm{1}$ [30], сопровождается выделением одной молекулы воды 3. Схема реакции построения кластера приведена на рисунке 1.

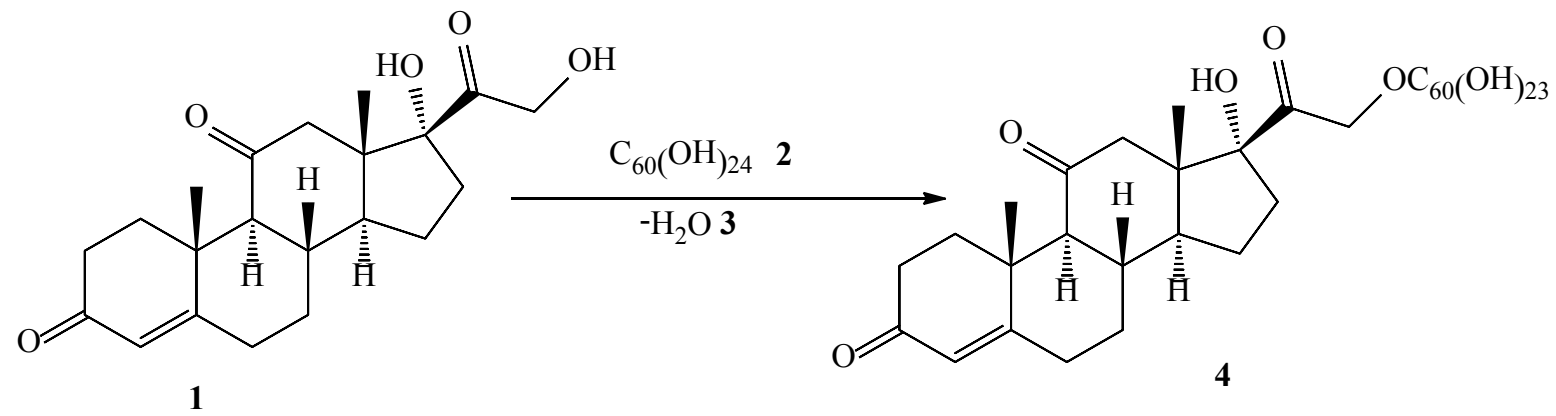

Рисунок 1. - Схема реакции построения кластера $(8 S, 9 S, 10 R, 13 S, 14 S, 17 R)-17$-гидрокси17-(2-фуллеренол[ $\left.\mathrm{C}_{60}(\mathrm{OH})_{23}\right]$ оксиацетил)-10,13-диметил-1,2,6,7,8,9,12,14,15,16декагидроциклопента $[a]$ фенантрен-3,11-диона) 4

В результате проведенного квантовохимического моделирования процесса построения кортизонофуллеренолового кластера 4 и расчета по формуле (1) теплового эффекта процесса установлено, что образование кластера 4 из исходных компонентов происходит с понижением полной энергии системы на 77,94 кДж/моль (таблица 1).

Таблица 1. - Полные энергии систем (E, a.e.), энергии высших занятых атомных и молекулярных орбиталей (B3MO, eV) и нижних вакантных атомных и молекулярных орбиталей $(\mathrm{HBMO}, \mathrm{eV})$, дипольные моменты $(D$, Дб) соединений 1-8, вычисленные в вакууме

\begin{tabular}{|l|c|c|c|c|}
\hline № соединения * & $E$, a.e. & B3MO, eV & HВMO, eV & $D$, Дб \\
\hline $\mathbf{1}$ & $-1192,31715469$ & $-5,162$ & $-2,276$ & 6,24 \\
\hline $\mathbf{2}$ & $-4103,14701248$ & $-5,421$ & $-3,568$ & 2,17 \\
\hline $\mathbf{3}$ & $-76,36045574$ & $-6,320$ & 0,815 & 1,97 \\
\hline $\mathbf{4}$ & $-5219,07402666$ & $-5,506$ & $-3,938$ & 9,29 \\
\hline $\mathbf{5}$ & $-288,79669325$ & $-7,854$ & $-0,696$ & 0 \\
\hline $\mathbf{6}$ & $-5507,91134423$ & - & - & 5,09 \\
\hline $\mathbf{7}$ & $-5507,90628875$ & $-5,287$ & $-3,606$ & 9,68 \\
\hline $\mathbf{8}$ & $-5796,77755374$ & $-5,315$ & $-3,619$ & 10,14 \\
\hline
\end{tabular}

Примечание:* 1 - кортизон; 2 - симметричный тетраэйкозофуллеренол; $\mathbf{3}$ - вода; 4-(8S,9S,10R,13S,14S,17R)-17-гидрокси-17-(2-фуллеренол[ $\left.\mathrm{C}_{60}(\mathrm{OH})_{23}\right]$ оксиацетил)-10,13-диметил$1,2,6,7,8,9,12,14,15,16$-декагидроциклопента[a]фенантрен-3,11-дион); 5 - атом радона ${ }^{222} \mathrm{Rn}$; 6 - атом ${ }^{222} \mathrm{Rn}$ снаружи кластера; 7 - атом ${ }^{222} \mathrm{Rn}$ внутри кластера; 8 - два атома ${ }^{222} \mathrm{Rn}$, снаружи и внутри.

В таблице 1 также приведены полные энергии систем $(E)$, вычисленные для соединений методом DFT, и дипольные моменты $(D)$ (полная энергия молекулы есть сумма электронной энергии и энергии отталкивания ядер, выраженная в атомных единицах Хартри).

При образовании кортизонофуллереноловых кластеров с радоном 5 возможны три состояния изучаемой системы (молекулярных ансамбля):

- молекулярный ансамбль 6 - атом радона 5 расположен снаружи кортизонофуллеренолового кластера 4;

- молекулярный ансамбль 7 - атом радона 5 находится внутри фуллереновой сферы 2;

- молекулярный ансамбль 8 - два ато- 
ма радона 5 находятся как внутри, так и снаружи кластера.

Результаты проведенных по формулам
(2), (3) $[25,26]$ квантово-химических расчетов энергии взаимодействия $\left(\Delta E_{I n t}\right)$ для кластеров 6-8 приведены в таблице 2 .

Таблица 2. - Оценка энергии образования кластера 4 из исходных компонентов и взаимодействия «пустого» кластера 4 с атомами радона в кластерах 6-8 $\left(\Delta E_{I n t}\right)$

\begin{tabular}{|l|c|c|}
\hline № соединения & $\Delta E_{\text {Int. }}$, а.е. (Хартри) & $\Delta E_{\text {Int. }}$, кДж/моль \\
\hline $\mathbf{4}$ & 0,02968477 & 77,94 \\
\hline $\mathbf{6}$ & $-0,04062432$ & $-106,66$ \\
\hline $\mathbf{7}$ & $-0,03556884$ & $-93,39$ \\
\hline $\mathbf{8}$ & $-0,11014058$ & $-289,17$ \\
\hline
\end{tabular}

Примечание: обозначения такие же, как в таблице 1.

Расчеты, выполненные по формулам (2) и (3), показывают устойчивость - понижение или повышение полной энергии системы - при гипотетическом «растворении» 1 или 2 моль атомов ${ }^{222} \mathrm{Rn} 5$ в 1 моль кортизонофуллеренола 4. В данном случае создание молекулярных ансамблей 6-8 из исходных компонентов происходит с выделением от 93,39 до 289,17 кДж/моль, что, по-видимому, обусловлено легкостью поляризации $4 f^{14} 5 d^{10}$-электронов атома радона 5 и возможностью образования соединений включения (клатратов) с кортизонофуллереноловым конъюгатом 4. Этот вывод следует и из анализа величин энергий высших занятых и нижних вакантных атомных или молекулярных орбиталей (ВЗМО и НВМО, таблица 1) этих веществ (4 и 5). Их значения достаточно близки.

Данные расчеты позволяют оценить зависимость биологической активности моделируемых соединений от их состава и электронной структуры. Как показано в работе [27], одной из характеристик биологической активности молекул является их реакционная способность, которая, в свою очередь, является характеристикой относительной химической активности молекул. Причем увеличение реакцион- ной способности изучаемого соединения может приводить к увеличению его химической и биологической активности. Для оценки реакционной способности, и соответственно, и биологической активности в работе [27] использовалась методология теории граничных молекулярных орбиталей (ГМО), разработанная К. Фукуи. Согласно концепции ГМО, протекание химических реакций и взаимодействие с биологическими мембранами клеток определяется разностями энергий между высшей занятой и низшей свободной молекулярными орбиталями исследуемых соединений (ВЗМО и НСМО, таблица 1). Чем эта величина меньше, тем более реакционноспособным является изучаемое соединение.

В соответствии с теорией ГМО [27] ключевые характеристики молекул - это величины $\Delta E_{\Gamma м о}$, которые рассчитывают по формулам (4) и (5) с использованием данных таблицы 1. В таблице 3 представлены разности энергий $\Delta E_{\Gamma м о}$, рассчитанные по формулам (4) и (5) и представленные в электронвольтах $(\mathrm{eV})$ и в джоулях $\Delta E_{Г м о}$, Дж $10^{-19}$ (для одиночной молекулы); и затем для наглядности пересчитанные путем умножения на число Авогадро для

Таблица 3. - Разности энергий ВЗАО и НВАО радона 5 и ВЗМО и НВМО соединений 1 , $2,4,6-8$ (для наглядности представлены в порядке их убывания)

\begin{tabular}{|c|c|c|c|c|}
\hline № соединения & $\Delta E_{\Gamma M O}, \mathrm{eV}$ & 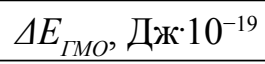 & $\Delta E_{\Gamma \text { Гмоло.) }}$, кДж/моль & $D$, Дб \\
\hline 5 & 7,158 & 11,468 & 690,603 & 0 \\
\hline 1 & 2,886 & 4,624 & 278,457 & 6,24 \\
\hline 2 & 1,853 & 3,323 & 200,111 & 2,17 \\
\hline 6 & 1,704 & 2,730 & 164,401 & 5,09 \\
\hline 8 & 1,696 & 2,717 & 163,016 & 10,14 \\
\hline 7 & 1,681 & 2,693 & 162,172 & 9,68 \\
\hline 4 & 1,568 & 2,512 & 151,273 & 9,29 \\
\hline
\end{tabular}

Примечание: обозначения такие же, как в таблице 1. 
1 моль атомов или 1 моль молекул изуча-

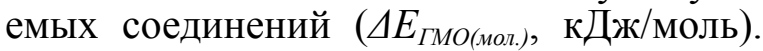
Последнее значение имеет следующий физический смысл - это энергия, необходимая для перехода 1 электрона с верхней занятой на нижнюю вакантную атомную или молекулярную орбиталь для 1 моль атомов 5 или 1 моль молекул соединений 1, 2, 6-8. Значение 1 моль определяется через число Авогадро: один моль - это количество вещества, содержащее $6,022140857(74) \cdot 10^{23}$ частиц (атомов, молекул, ионов, протонов, нейтронов, $\alpha$-частиц, электронов или любых других объектов).

Данные, приведенные в таблице 3, демонстрируют корректность метода ГМО. Наиболее слабую реакционную способность и, следовательно, наименьшую возможность взаимодействовать с органеллами клеток-мишеней (сродство или аффинность к биологическим мембранам, ферментам и т.д.) ожидаемо проявляет сам инертный газ радон 5. Максимальную способность взаимодействовать с биологическими объектами проявляет кортизонофуллереноловый «пустой» кластер 4, превосходя в этом отношении исходные компоненты, взятые для его построения кортизон 1 и фуллеренол 2. Реакционная способность радоносодержащих класте- ров 6-8 очень мало отличается от «пустого» кластера 4: ее снижение составляет всего около 6-8\%. Дипольные моменты соединений $1,2,4,6-8$ указывают на возрастание полярности изучаемых соединений при переходе от неполярного радона 5 (0 Дб) к кластерам 4, 7, 8 (9,29-10,14 Дб).

На рисунке 2 представлена модель строения кортизонофуллереноловых кластеров: 4 - «пустой» кортизонофуллереноловый кластер (Е и М отсутствуют), 6 атом радона снаружи кластера $\left(\mathrm{E}={ }^{222} \mathrm{Rn}\right.$, М отсутствует); 7 - атом радона внутри фуллереноловой сферы (Е отсутствует, $\left.\mathrm{M}={ }^{222} \mathrm{Rn}\right) ; 8$ - два атома радона снаружи и внутри кластера $\left(\mathrm{E}=\mathrm{M}={ }^{222} \mathrm{Rn}\right)$.

Для введения (интеркаляции) атомов радона во внутреннюю полость фуллереноловой сферы могут быть применены технологии высоких и сверхвысоких давлений (вплоть до 400 тысяч атмосфер или более). Сжатие - один из самых сильных инструментов воздействия на твердое тело. С его помощью изменяются свойства твердых тел благодаря изменению расстояний между атомами. Эти расстояния можно менять и другими способами, например, повышая или понижая температуру. Однако температурные воздействия ограничены температурой необратимой

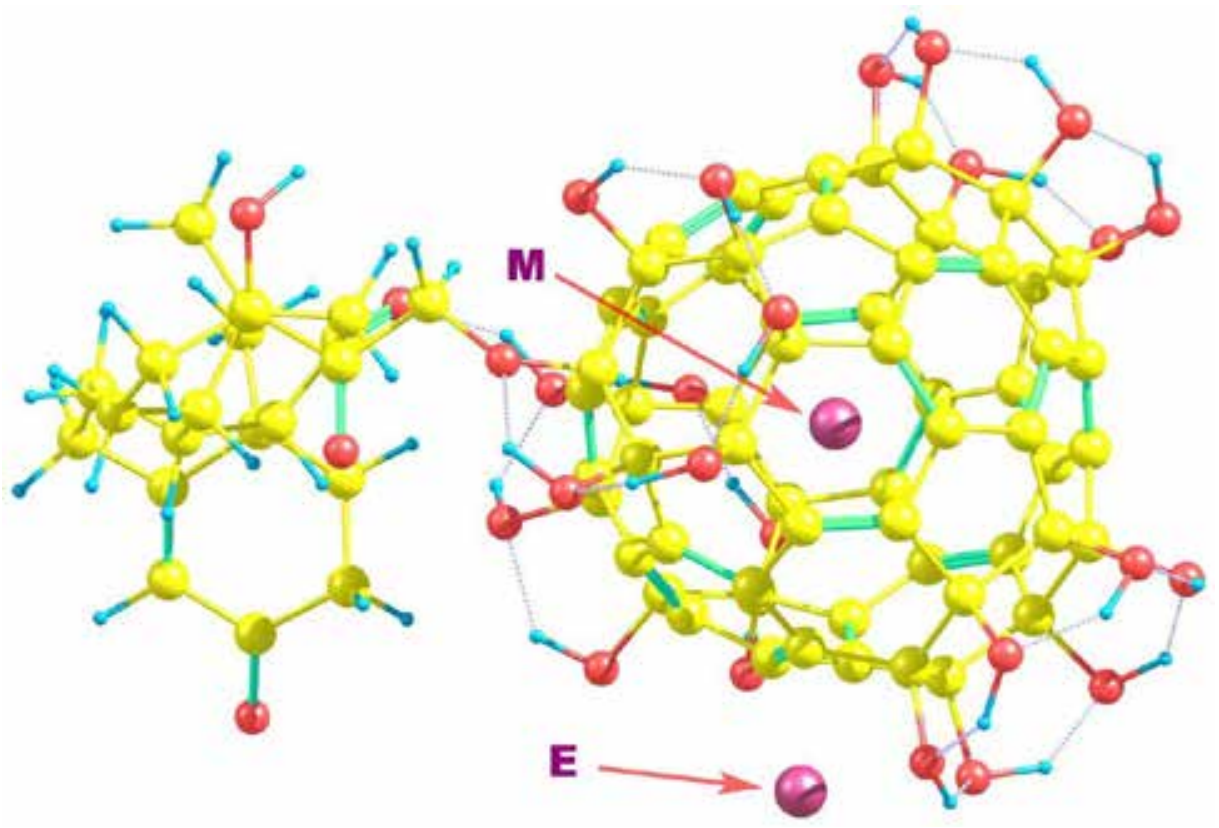

Обозначения: 4 - «пустой» кортизонофуллереноловый кластер (Е и М отсутствуют), 6 - атом радона снаружи кластера $\left(\mathrm{E}={ }^{222} \mathrm{Rn}, \mathrm{M}\right.$ отсутствует); 7 - атом радона внутри фуллереноловой сферы (Е отсутствует, $\left.\mathrm{M}={ }^{222} \mathrm{Rn}\right) ; 8$ - два атома радона снаружи и внутри кластера $\left(\mathrm{E}=\mathrm{M}={ }^{222} \mathrm{Rn}\right)$.

Рисунок 2. - Модель строения кортизонофуллереноловых кластеров 4, 6-8 
деструкции вещества, на которое собираются воздействовать нагревом. При подобных воздействиях вандерваальсовы радиусы атомов уменьшаются на $30-40 \%$ и атомы радона должны легко «впрессоваться» во внутренние полости фуллеренола [31].

\section{ЗАКЛЮЧЕНИЕ}

Радоносодержащие кортизонофуллереноловые агенты терапии онкологических заболеваний могут оказаться перспективными для разработки на их основе новых методов адресной доставки медицинских радионуклидных препаратов в целевые клетки-мишени без поражения здоровых тканей и органов. Из полученных данных об устойчивости исследованных методом квантово-химического DFT-моделирования кластеров можно сделать выводы о возможности их практического получения.

Работа выполнена при финансовой поддержке ГПНИ «Междисциилинарные исследования и новые зарождающчиеся технологии (Конвергенциия-2025)».

\section{SUMMARY}

E. A. Dikusar, A. L. Pushkarchuk,

T. V. Bezyazychnaya, E. A. Akishina, A. G. Soldatov, S. A. Kuten, S. G. Stepin, A. P. Nizovtsev, S. Ya. Kilin, L. F. Babichev, V. I. Potkin

\section{PROSPECTS FOR CREATING RADON CONTAINING AGENTS \\ IN RADIONUCLIDE THERAPY}

One of the methods for treating oncological diseases is application of short-lived radionuclides. Existing risks of intoxication and radioactive contamination can be eliminated by introduction of a short-lived radon isotope into a hard strong fullerene coat. To increase the effectiveness of these drugs it is promising to introduce structural fragments of amino acids, peptides, steroid hormones, nucleic bases or heterocyclic compounds being able to accumulate exactly in tumoral but not in healthy tissues of the body. The article presents data on quantum-chemical simulation of electronic structure and thermodynamic stability of fullerenol radon-containing agents of radionuclide therapy on the example of a model cortisonefullerenol bioconjugate.

Keywords: DFT-simulation, radon isotope, cortisone, fullerenol clusters, agents for the treatment of tumoral neoplasms.

\section{ЛИТЕРАТУРА}

1. Овчинников, В. А. Современные методы лучевого лечения онкологических больных / В. А. Овчинников, К. Н. Угляница, В. Н. Волков // Журн. Гродненского гос. мед. ун-та. 2010. - № 1. - C. 93-97.

2. Handbook of Radiationtherapy Physics: Theory and Practice / ed.: P. Mayles, A. Nahum, J. C. Rosenwald. - New York; London: Taylon \& Francis, 2007. - 1450 p.

3. Hosmane, N. S. Boron and Gadolinium Neutron Capture Therapy for Cancer Treatment / N. S. Hosmane, J. A. Maquire, Y. Zhu. - [S. 1.]: World Scientific Publ. Co., 2012. - 300 p.

4. Оценка возможности использования эндоэдрических радон-222-содержащих производных бакминстерфуллеренов С60 и С80 в качестве нанороботов-истребителей опухолевых новообразований / Е. А. Дикусар [и др.] // Вестн. фармации. - 2012. - № 4. - С. 102-105.

5. Эндоэдрические радон-222-содержащие производные бакминстерфуллеренов С60 и С80 / Е. А. Дикусар [и др.] // Углеродные наночастицы в конденсированных средах: сб. науч. ст. - Минск: Ин-т тепло- и массообмена им. А. В. Лыкова НАН Беларуси, 2013. C. 246-250.

6. Quantum-Chemistry Projecting of the Radionuclide Carborane and Fullerene Nano-Cluster Agents for Diagnostics and Therapy of Oncological Diseases / E. A. Dikusar [et al.] // Physics, Chemistry and Application of Nanostructures: proc. of International Conf. Nanomeeting - 2013: reviews and short notes, Minsk, Belarus, 24-27 may 2013 y. / ed.: V. E. Borisenko [et al.]. Minsk, 2013. - P. 324-327.

7. Квантово-химическое проектирование радионуклидных агентов для диагностики и терапии опухолевых новообразований / Е. А. Дикусар [и др.] // Вестн. Тверского гос. ун-та. Сер. Физика. - 2013. - № 21. - С. 69-81.

8. Quantum chemical designing of endohedral containing $\mathrm{Po}^{210}$ derivatives of buckminsterfullerene $\mathrm{C}_{60}-\mathrm{C}_{80}$ for development of radionuclide nanosized agents for cancer therapy / E. A. Dikusar [et al.] // Nonlinear Dynamics and Applications: proc. of the 21-th Annual Seminar NPCS'2014, Minsk, 20-23 may 2014 y. / ed.: L. F. Babichev, V. I. Kuvshinov, V. A. Shaparau. Minsk, 2014. - Vol. 20. - P. 50-55.

9. Компьютерное проектирование методом DFT эндоэдрических $\mathrm{Po}^{210}$-содержащих фуллеренов $\mathrm{C}_{60}-\mathrm{C}_{80}$ в качестве радионуклидных агентов терапии онкологических заболеваний / Е. А. Дикусар [и др.] // Наноструктуры в конденсированных средах: сб. науч. ст. Минск: Ин-т тепло- и массообмена им. А. В. Лыкова НАН Беларуси, 2014. - С. 275-280.

10. Квантово-химическое проектирование эндоэдрических индийсодержащих про- 
изводных бакминстерфуллеренов $\mathrm{C}_{60}$ и $\mathrm{C}_{70}$ для разработки радионуклидных наноразмерных агентов-истребителей опухолевых новообразований / Е. А. Дикусар [и др.] // Физико-химические аспекты изучения кластеров, наноструктур и наноматериалов: межвуз. сб. науч. тр. / под общ. ред. В. М. Самсонова, Н. Ю. Сдобнякова. - Тверь: Тверской гос. ун-т, 2014. - Вып. 6. - С. 105-115.

11. Дикусар, Е. А. Квантово-химическое моделирование структуры холестеринсодержащих радионуклидных агентов терапии онкологических заболеваний / Е. А. Дикусар // Междунар. науч. ин-т «Educatio». - 2015. № 2, ч. 4. - С. 72-75.

12. Квантово-химическое проектирование эндоэдрических бакминстерфуллереновых кластеров на основе бисфуллеренола для создания радионуклидных агентов терапии онкологических заболеваний / Е. А. Дикусар [и др.] // Междунар. науч.-исследоват. журн. - 2015. № 5, ч. 1. - С. 35-41.

13. Квантово-химическое проектирование эндоэдрических бисбакминстерфуллереновых кластерных систем для разработки нанокапсулированных радионуклидных агентов терапии онкологических заболеваний / Е. А. Дикусар [и др.] // Физико-химические аспекты изучения кластеров, наноструктур и наноматериалов: межвуз. сб. науч. тр. / под общ. ред. В. М. Самсонова, Н. Ю. Сдобнякова. - Тверь: Тверской гос. ун-т, 2015. - Вып. 7. - С. 193-207.

14. Квантово-химическое проектирование эндоэдрических бакминстерфуллереновых кластерных систем для создания радионуклидных агентов терапии онкологических заболеваний / Е. А. Дикусар [и др.] // Вестн. фармации. - 2015. - № 4. - С. 51-59.

15. Quantum chemical designing of cholesterol containing radionuclide anticancer agents / E. A. Dikusar [et al.] // Functional Materials. 2016. - Vol. 23, N 2. - P. 274-278.

16. Quantum Chemical Designing of Cholesterol and Estrone Containing Radionuclide Cancer-Fighting Agents / E. A. Dikusar [et al.] // Весці НАН Беларусі. Сер. хім. навук. - 2016. № 3. - C. 68-69.

17. Квантово-химическое моделирование эстронсодержащих бисфуллереноловых радионуклидных агентов терапии онкологических заболеваний / Е.А. Дикусар [и др.] // Наноструктуры в конденсированных средах: сб. науч. ст. - Минск: Ин-т тепло- и массообмена им. А. В. Лыкова НАН Беларуси, 2016. C. $71-76$.

18. Квантово-химическое моделирование строения эстронсодержащих бисфуллереноловых радионуклидных агентов терапии онкологических заболеваний / Е. А. Дикусар [и др.] // Физико-химические аспекты изучения кластеров, наноструктур и наноматериалов: межвуз. сб. науч. тр. / под общ. ред. В. М. Самсонова, Н. Ю. Сдобнякова. - Тверь: Твер. гос. ун-т, 2016. - Вып. 8. - С. 110-118.

19. Квантово-химическое моделирование строения метотрексатсодержащих фуллереноловых радионуклидных агентов терапии онкологических заболеваний / Е. А. Дикусар [и др.]// Вестн. фармации. - 2017. - № 1. - С. 52-56.

20. DFT Simulation of Methotrexate Fullerenol Radionuclide Agents of Cancer Therapy E. A. Dikusar [et al.] // Nonlinear Dynamics and Applications. - Минск: Право и экономика, 2017. Vol. 23. - P. 143-149.

21. Квантово-химическое моделирование метотрексат-фуллереноловых радионуклидных агентов терапии онкологических заболеваний / Е. А. Дикусар [и др.] // Весці НАН Беларусі. Сер. хім. навук. - 2019. - Т. 55, № 2. - C. 163-170.

22. Квантово-химическое моделирование эндоэдрических производных фуллеренолокортизона / Е. А. Дикусар [и др.] // Фуллерены и наноструктуры в конденсированных средах: сб. науч. ст. - Минск: Ин-т тепло- и массообмена им. А. В. Лыкова НАН Беларуси, 2021. C. 64-67.

23. Физиотерапия: нац. рук. / под ред. Г. Н. Пономаренко - Москва: ГЭОТАР-Медиа, 2009. $-864 \mathrm{c}$.

24. The ORCA quantum chemistry program package / F. Neese [et al.] // The J. of Chem. Physics. - 2020. - Vol. 152, N 22. - Art. 224108.

25. Sandler, S. I. Chemical, biochemical, and engineering thermodynamics / S. I. Sandler. New York: John Wiley \& Sons, 2017. - 1040 p.

26. Demerel, Y. Nonequilibrium thermodynamic6: Transport and rate processes in physical, chemical and biological systems / Y. Demerel. 3rd ed. - Amsterdam ; Oxford: Elsevier Science, 2014. - $792 \mathrm{p}$.

27. Putz, M. V. DFT chemical reactivity driven by biological activity: applications for the toxicological fate of chlorinated PAHs / M. V. Putz, A. M. Putz // Applications of Density Functional Theory to Biological and Bioinorganic Chemistry / ed.: M. V. Putz, M. P. Mingos. - Berlin: Springer Link, 2013. - P. 181-231.

28. Тетракозагидроксибакминстерфуллеренол - реагент будущего / Е. А. Дикусар [и др.] // Химические реактивы, реагенты и процессы малотоннажной химии: тез. докл. XXXI Междунар. науч.-техн. конф. «Реактив-2018», Минск, 2-4 окт. 2018 г. / редкол.: В. Е. Агабеков, В. А. Хрипач, Ж. В. Игнатович. - Минск: Беларуская навука, 2018. - С. 22.

29. Antitumor Activity and Potential Mechanism of Novel Fullerene Derivative Nanoparticles / L. Ye [et al.] // Molecules. - 2021. - Vol. 26, N 11. - Art. 3252.

30.Физер, Л. Стероиды / Л. Физер, М. Физер. - Москва: Мир, 1964. - 982 с. 
31. Sundqvist, B. Fullerenes under high pressure/B. Sundqvist//Fullerenes: chemistry, physics, and technology / ed: K. M. Kadish, R. S. Ruoff. New York: Wiley-Interscience, 2000. - 984 p.

\section{REFERENCES}

1. Ovchinnikov VA, Uglianitsa KN, Volkov VN. Modern methods of radiation treatment of cancer patients. Zhurn Grodnenskogo gos med un-ta. 2010;(1):93-7. (In Russ.)

2. Mayles P, Nahum A, Rosenwald JC, editors. Handbook of Radiationtherapy Physics: Theory and Practice. New York, USA: Taylon \& Francis; 2007. $1450 \mathrm{p}$

3. Hosmane NS, Maquire JF, Zhu Y. Boron and Gadolinium Neutron Capture Therapy for Cancer Treatment. [place unknown]: World Scientific Publ Co; 2012. 300 p.

4. Dikusar EA, Zelenkovskii VM, Pushkarchuk AL, Potkin VI, Rudakov DA, Soldatov AG, i dr. Evaluation of the possibility of using endohedral radon-222-containing derivatives of buckminsterfullerenes $\mathrm{C} 60$ and C80 as nanorobots - destroyers of tumor neoplasms. Vestn farmatsii. 2012;(4):102-5. (In Russ.)

5. Dikusar EA, Zelenkovskii VM, Pushkarchuk AL, Potkin VI, Rudakov DA, Soldatov AG i dr. Endohedral radon-222containing derivatives of buckminsterfullerenes C60 and C80. V: Uglerodnye nanochastitsy v kondensirovannykh sredakh: sb nauch st. Minsk, RB: In-t teplo- i massoobmena im AV Lykova NAN Belarusi; 2013. s. 246-50. (In Russ.)

6. Dikusar EA, Zelenkovski VM, Potkin VI, Rudakov DA, Kuten SA, Soldatov AG. QuantumChemistry Projecting of the Radionuclide Carborane and Fullerene Nano-Cluster Agents for Diagnostics and Therapy of Oncological Diseases. In: Borisenko VE, Gaponenko SV, Gurin VS, Kam $\mathrm{CH}$, editors. Physics, Chemistry and Application of Nanostructures. Proceedings of International Conference Nanomeeting - 2013 : reviews and short notes; 2013 May 24-27; Minsk, Belarus. Minsk, RB; 2013. p. 324-7

7. Dikusar EA, Zelenkovskii VM, Pushkarchuk AL, Rudakov DA, Soldatov AG, Kuten' SA. Quantum-chemical design of radionuclide agents for the diagnosis and therapy of tumor neoplasms. Vestn Tverskogo gos un-ta. Ser Fizika. 2013;(21):69-81. (In Russ.)

8. Dikusar EA, Zelenkovskii VM, Pushkarchuk AL, Kilin SYa, Soldatov AG, Kuten SA et al. Quantum chemical designing of endohedral containing $\mathrm{Po}^{210}$ derivatives of buckminsterfullerene $\mathrm{C}_{60}-\mathrm{C}_{80}$ for development of radionuclide nanosized agents for cancer therapy. In: Babichev LF, Kuvshinov VI, Shaparau VA, editors. Nonlinear Dynamics and Applications. Proceedings of the 21-th Annual Seminar NPCS'2014; 2014 May 20-23; Minsk, Belarus.
Minsk, RB; 2014. Vol 20. p. 50-5

9. Dikusar EA, Pushkarchuk AL, Zelenkovskii VM, Kilin SYa, Kuten' SA, Soldatov AG i dr. DFT computer-aided design of endohedral Po210-containing fullerenes C60 C80 as radionuclide agents in the therapy of oncological diseases. V: Nanostruktury V kondensirovannykh sredakh: sb nauch st. Minsk, RB: In-t teplo- i massoobmena im AV Lykova NAN Belarusi; 2014. s. 275-80. (In Russ.)

10. DikusarEA, Zelenkovskii VM, Pushkarchuk AL, Potkin VI, Rudakov DA, Kilin SYa i dr. Quantum-chemical design of endohedral indiumcontaining derivatives of Buckminsterfullerenes C60 and C70 for the development of nanosized radionuclide agents-destroyers of tumor neoplasms. V: Samsonov VM, Sdobniakov NIu, redaktory. Fiziko-khimicheskie aspekty izucheniia klasterov, nanostruktur i nanomaterialov: mezhvuz sb nauch tr. Tver', RF: Tverskoi gos un-t; 2014. Vyp 6. s. 10515. (In Russ.)

11. Dikusar EA. Quantum-chemical modeling of the structure of cholesterolcontaining radionuclide agents for the treatment of oncological diseases. Mezhdunar nauch in-t «Educatio». 2015;(2 Ch 4):72-5. (In Russ.)

12. Dikusar EA, Zelenkovskii VM, Pushkarchuk AL, Potkin VI, Kilin SYa, Soldatov AG i dr. Quantum-chemical design of endohedral buckminsterfullerene clusters based on bisfullerenol for the creation of radionuclide agents for the therapy of oncological diseases. Mezhdunar nauch-issledovat zhurn. 2015;(5 Ch 1):35-41. (In Russ.)

13. Dikusar EA, Pushkarchuk AL, Potkin VI, Kilin SYa, Soldatov AG, Kuten' SA i dr. Quantum-chemical design of endohedral bisbacminsterfullerene cluster systems for the development of nanocapsulated radionuclide agents for the treatment of oncological diseases. V: Samsonov VM, Sdobniakov NIu, redaktory. Fiziko-khimicheskie aspekty izucheniia klasterov, nanostruktur i nanomaterialov: mezhvuz sb nauch tr. Tver', RF: Tverskoi gos un-t; 2015. Vyp 7. s. 193-207. (In Russ.)

14. Dikusar EA, Zelenkovskii VM, Pushkarchuk AL, Potkin VI, Kilin SYa, Soldatov AG i dr. Quantum-chemical design of endohedral buckminsterfullerene cluster systems for the creation of radionuclide agents for the therapy of oncological diseases. Vestn farmatsii. 2015;(4):51-9. (In Russ.)

15. Dikusar EA, Zelenkovskii VM, Pushkarchuk AL, Rudakov DA, Potkin VI, Kilin SYa et al. Quantum chemical designing of cholesterol containing radionuclide anticancer agents. Functional Materials. 2016;23(2):274-8

16. Dikusar EA, Pushkarchuk AL, Rudakov DA, Potkin VI, Kozlov NG, Kilin SYa et al. Quantum Chemical Designing of Cholesterol and Estrone Containing Radionuclide Cancer- 
Fighting Agents. Vestsi NAN Belarusi. Ser khim navuk. 2016;(3):68-9

17. Dikusar EA, Potkin VI, Pushkarchuk AL, Bez»iazychnaia TV, Soldatov AG, Kuten' SA i dr. Quantum-chemical modeling of estronecontaining bisfullerenol radionuclide agents for the treatment of oncological diseases. V: Nanostruktury v kondensirovannykh sredakh: sb nauch st. Minsk, RB: In-t teplo- i massoobmena im AV Lykova NAN Belarusi; 2016. s. 71-6. (In Russ.)

18. Dikusar EA, Pushkarchuk AL, Bez»iazychnaia TV, Soldatov AG, Kuten' SA. Quantum-chemical modeling of the structure of estrone-containing bisfullerenol radionuclide agents for the treatment of oncological diseases. V: Samsonov VM, Sdobniakov NIu, redaktory. Fiziko-khimicheskie aspekty izucheniia klasterov, nanostruktur i nanomaterialov: mezhvuz sb nauch tr. Tver', RF: Tverskoi gos un-t; 2016. Vyp 8. s. 110-8. (In Russ.)

19. Dikusar EA, Pushkarchuk AL, Soldatov AG, Kuten' SA, Stepin SG. Quantum-chemical modeling of the structure of methotrexatecontaining fullerenol radionuclide agents for the treatment of oncological diseases. Vestn farmatsii. 2017;(1):52-6. (In Russ.)

20. Dikusar EA, Bezyazychnaya TV, Potkin VI, Kosandrovich EG, Pushkarchuk AL, Soldatov AG et al. DFT Simulation of Methotrexate Fullerenol Radionuclide Agents of Cancer Therapy. In: Nonlinear Dynamics and Applications. Minsk, RB: Pravo i ekonomika; 2017. Vol 23. p. 143-9

21. Dikusar EA, Pushkarchuk AL, Bez»iazychnaia TV, Potkin VI, Soldatov AG, Kuten' SA i dr. Quantum-chemical modeling of methotrexate-fullerenol radionuclide agents for the treatment of oncological diseases. Vestsi NAN Belarusi. Ser khim navuk. 2019;55(2):163-70. (In Russ.)

22. Dikusar EA, Pushkarchuk AL, Bez»iazychnaia TV, Potkin VI, Soldatov AG, Kuten' SA i dr. Quantum Chemical Modeling of Endohedral Fullerenolocortisone Derivatives. V: Fullereny i nanostruktury $\mathrm{v}$ kondensirovannykh sredakh: sb nauch st. Minsk, RB: In-t teplo- i massoobmena im AV Lykova NAN Belarusi; 2021. s. 64-7. (In Russ.)

23. Ponomarenko GN, redaktor. Physiotherapy: nats ruk. Moskva, RF: GEOTARMedia; 2009. 864 s. (In Russ.)
24. Neese F, Wennmohs F, Becker U, Riplinger $C$. The ORCA quantum chemistry program package. J. Chem. Phys. 2020;152(22 art 224108). doi: 10.1063/5.0004608

25. Sandler SI. Chemical, biochemical, and engineering thermodynamics. New York, USA: John Wiley \& Sons; 2017. $1040 \mathrm{p}$

26. Demerel Y. Nonequilibrium thermodynamics: Transport and rate processes in physical, chemical and biological systems. 3rd ed. Amsterdam, Netherlands: Elsevier Science; 2014. $792 \mathrm{p}$

27. Putz MV, Putz AM. DFT chemical reactivity driven by biological activity: applications for the toxicological fate of chlorinated PAHs. In: Putz MV, Mingos MP, editors. Applications of Density Functional Theory to Biological and Bioinorganic Chemistry. Berlin, Germany: Springer Link; 2013. p. 181-231

28. Dikusar EA, Pushkarchuk AL, Bez»iazychnaia TV, Kosandrovich EG, Soldatov AG, Kuten' SA i dr. Tetracosahydroxybakminster fullerenol - the reagent of the future. V: Agabekov VE, Khripach VA, Ignatovich $\mathrm{ZhV}$, redkollegiia. Khimicheskie reaktivy, reagenty i protsessy malotonnazhnoi khimii. Tezisy dokladov XXXI Mezhdunarodnoi nauchno-tekhnicheskoi konferentsii «Reaktiv-2018»; 2018 Okt 2-4; Minsk, Belarus'. Minsk, RB: Belaruskaia navuka; 2018. s. 22. (In Russ.)

29. Ye L, Kollie L, Liu X, Guo W, Ying X, Zhu $\mathrm{J}$ et al. Antitumor Activity and Potential Mechanism of Novel Fullerene Derivative Nanoparticles. Molecules. 2021;26(11 art 3252). doi: 10.3390/molecules26113252

30. Fizer L, Fizer M. Steroids. Moskva, RF: Mir; 1964. 982 s. (In Russ.)

31. Sundqvist B. Fullerenes under high pressure. In: Kadish KM, Ruoff RS, editors. Fullerenes: chemistry, physics, and technology. New York, USA: Wiley-Interscience; 2000. 984 p

\section{Адрес для корреспонденци: \\ 220072, Республика Беларусь, \\ г. Минск, ул. Сурганова, 13, \\ Институт физико-органической химии \\ Национальной академии наук Беларуси, тел.: +375-17-2841600, моб. +375-29-6228644, e-mail:dikusar@ifoch.bas-net.by, \\ Дикусар Е. A.}

Поступила 20.09.2021 г. 